Cognitive-behavior intervention group counseling manual for reducing adolescents' career indecision

Datu, Jesus Alfonso $\bowtie$

De La Salle University, Manila, Philippines (jess.datu@yahoo.com)

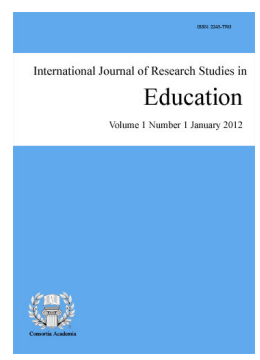

Accepted: 3 September 2012

ISSN: 2243-7703 Online ISSN: 2243-7711

OPEN ACCESS

\title{
Abstract
}

The current manual is purported to provide an empirical guide in facilitating a group intervention that will address career indecision among adolescents. It utilized Cognitive-Behavioral Therapy as the major framework of the treatment protocol. Prior to the group facilitation, prospective members will be screened through an interview and Career Decision Profile. It consists of six sessions (one and a half hour every session) which will be executed on a weekly basis. With the intention of modifying negative beliefs that the members hold about themselves in relation to career decision-making, specific activities and processing procedures were charted each session that ranged from individual cognitive exercises to dyadic behavioral role-plays. Each session will be monitored by the group counselor via group case notes to properly document therapeutic encounters which is essential in achieving the intended outcomes. At the end of the group intervention, members will be assessed through group feedback and administering of Career Decision Profile to look at the positive changes on their levels of capabilities to make career decisions.

Keywords: adolescents; career indecision; Cognitive-Behavioral Therapy; group manual; therapeutic factors 


\section{Cognitive-behavior intervention group counseling manual for reducing adolescents' career indecision}

\section{Purpose}

The aim of this intervention is to provide a venue where individuals can maximize their potentials for occupational growth and development through series of activities such as identification and modification of dysfunctional beliefs, behavioral role plays, and recommended homework. The ultimate purpose of the group is to increase the members' adaptability to career-related and other concerns by equipping them with necessary cognitive and behavioral skills that will make them personally-efficacious without developing dependency to counselors and therapists.

\section{Theoretical framework}

Cognitive-Behavioral Therapy (CBT) has the widest pragmatic utility in clinical and counseling settings. A good number of practice-based evidences that highlight the efficacy of this therapeutic modality have been generated in previous studies. Among the line of inquiries that were addressed by cognitive-behavioral approach include anger management (Cox \& Harrison, 2008), depression (Marcotte, Levesque, \& Fortin, 2006; Fujisawa et al., 2010), post-disaster distress (Hamblen, Norris, Pietruszkiewicz, Gibson, Naturale, \& Louis, 2009), and obsessions and compulsions (Sica, Taylor, Arrindell, \& Sanavio, 2006). Hence, it is very apparent that CBT has gained reputation over the past few decades as can be demarcated on the empirical inquiries that investigated its ability to treat wide array of psychological concerns and psychopathologies.

In essence, cognitive behavioral therapy is one of the orientations that have rooted from the constructivist paradigm in addressing problems that are experienced by individuals. It zeroes in on the assertion that "people are active participants on the in the construction of their own reality" (Granvold, 1994, p. 5). That being said, it can be surmised that individuals are responsible for the emergence of emotional disturbances and psychological concerns. Though social, circumstantial and other factors play a crucial role in the behavioral patterns of an individual, the manner of giving rational interpretation about such variables are within his control. As such, this therapeutic philosophy views that an individual is capable of changing despite the presence of extrinsic impediments.

Maladaptive behaviors and emotions are induced by a dynamic interaction that exists in one's cognition, feeling and behaviors. CBT posits that even though cognitions do not solely cause dysfunction, they are inevitable part of it (Beck \& Weishar, 1989 as cited in Granvold, 1994). It is very apparent that cognition is the target domain of change. Specifically, the negative beliefs that individuals hold about specific events termed as "automatic thoughts" lead them to experience emotional disturbances. Through cognitive distortions (e.g. false generalization, polarized thinking, blaming), individuals learn to acquire negative views about themselves

In the same way as it is experienced in some clinical conditions like depression, anxiety, phobia, etc., lack of efficacy of individuals in choosing career is speculated to be an issue that can be addressed using cognitive-behavioral approach. Dysfunctional career thoughts appeared to be a crucial factor in making career decisions (Austin, Wagner, \& Dahl, 2003). For instance, in a study done by Dimakakou, Mylonas, Argyropoulou, and Tampouri (2012), dysfunctional career beliefs about career decision were negatively correlated with self-efficacy. In particular, adolescents were among the populace who are immediately burdened by career-decision making difficulties given that making career choices is a crucial developmental task that they usually engaged in. However, there is a marked dearth of literature that capitalized on the use of CBT in dealing with career-related issues of individuals, especially in the Philippine context. This seemed to be a concern that deserved to be given due attention as there is a gradual demand to move towards evidenced-base practice. In the 
absence of a theoretically-grounded intervention that will address hardships experienced by this populace in deciding for their occupational inclinations, it will be relatively difficult to meet desirable therapeutic outcomes which will make their lives more meaningful, substantial and satisfying.

Bearing such considerations in mind, this manual was crafted to develop a group intervention protocol that will further provide evidences on the effectiveness of CBT in making adolescents more decided about the career options that have undertaken. Despite its proven therapeutic worth on individual treatments, it is interesting to look at how CBT can facilitate changes on the lives of individuals when applied in groups since social support and sense of belongingness may be perceived by most people as important catalysts of psychological well-being. By doing so, career-related concerns of adolescents can be addressed in a time-efficient manner with reference a psychotherapeutic intervention that is highly grounded with empirical evidences.

\section{Group goals}

The objective of the intervention is to lead the members towards realization of their respective career paths despite the presence of various obstacles. Most of the activities are purported to make them aware about their negative beliefs about their career choices, how it will affect their career-related behaviors and what they can do to modify such negative thinking patterns. Specifically, the following goals are expected to be met at the end of the process:

1. To become aware of the dysfunctional thoughts that we hold about ourselves as it relates to career behaviors.

2. To recognize the role of dysfunctional beliefs in shaping career decision-making difficulties.

3. To modify negative beliefs about one's self in relation to career decision-making process.

4. To explore effective ways of becoming more decided about a specific career option.

5. To foster a supportive atmosphere where each member can share unique ways on how to modify negative beliefs that they hold about themselves.

6. To increase perceived capability to make a career decision.

7. To become more decided about one's career path.

8. To exercise more control in their lives.

\section{Pre-group screening and orientation}

The group is suitable for 6-8 members. It will be composed of six sessions, with approximately one and a half hour per session on a weekly basis. Given that the group's primary goal is to reduce the members' level of perceived inability and increase their perceived ability in making career choices, two procedures will be utilized to identify the levels of their perceived career indecision. Firstly, Career Decision Profile (CDP), a 16-item inventory that intends to gauge career indecision will be administered. Next to that will be an interview by the group facilitator that is purported to check if there is a good match between the profiles of the prospective members and the goals of the group.

Integrated in the interview will be an exploration of the counseling or psychiatric history and behavioral observations of the aspiring members to recognize if they were experiencing serious maladaptive behavioral conditions (e.g. major depressive disorder, paranoid personality disorder, agoraphobia, etc.) that will aid in assessing their possible inclusion or exclusion in the group. It must be noted that only adolescents who have been experiencing mild behavioral concerns especially, career decision-making difficulties will be accommodated. Prospective members who may be presenting serious psychological, emotional and social 
Datu, J. A.

concerns will be referred to other qualified counselors or psychologists for individual counseling.

\section{Outline for six group sessions}

\subsection{Session 1}

\subsubsection{Purpose}

The objective for the first session is to maximize opportunities for each member to know one another in the group. They will be encouraged to share the circumstance or thoughts that have lead them to join the group. The session is allotted as well for the group leader to discuss the things that the group can offer to its members, the responsibilities of each member and matters about confidentiality. Collective therapeutic rapport and alliance will be established to ensure that each member will have a positive outlook and trust in the group.

\subsubsection{Theme}

The first session's theme will revolve around how each member of the group describes their career indecision as rooting from their negative thinking patterns. Most of the career-related concerns that will possibly arise include difficulties in deciding for a specific career path due to parental, financial, personal and identity constraints.

\subsubsection{Activities}

In the first activity, introduction of the group facilitator and members will be executed. The members will be encouraged share the reasons that prompted them to join the group. The facilitator will pull out the common reasons that will be cited by the members which let them experience some sort of difficulties in making occupational decisions. Based on the answers of the members, the facilitator cites common causes of career indecision and defines it as can be traced from its conceptual and practical standpoints. Thereafter, each member will be asked to share the negative beliefs that they hold about themselves in relation to the career paths that they are planning to engage in or they have engaged in. For example, an Engineering student would be thinking that he will surely fail all the major subjects in the course given that he thinks that he is stupid in Math. This is one of the initial steps in the course of the intervention which will allow the members to assess their selves. Other members will be given an opportunity to share their thoughts about the negative beliefs each member would be brining in the group. The facilitator presents a mini-lecture on automatic thoughts and they would play a crucial role in learning maladaptive thinking, emotional and behavioral patterns. After the mini-lecture, each member is tasked to identify specific automatic thoughts that reinforce the difficulties they are facing in the career choices they have or they are planning to engage in. They will be asked to share their identified automatic thoughts. Lastly, the facilitator pulls out commonalities and differences among the identified automatic thoughts of the members. Necessary processes and events in the group are identified as well to guide the group in the succeeding sessions.

\subsubsection{Process}

As the initial session of this group intervention, the set of activities are used were tailored from one of the core elements in the facilitation of cognitive-behavioral oriented treatment approach. The importance of self-assessment is highlighted given that each member is asked to examine the negative thoughts they hold about themselves in relation to the career choices that they have undertaken or they are planning to pursue. Informing the members about the effects of automatic thoughts on their career behavioral patterns is an essential step to make them aware about how one's cognition can predispose them to experience negative emotional and psychological state that can be manifested in their behaviors. Each member can also learn from one another regarding the adverse effects of having negative belief systems on their lives. As such, sharing of the experiences from each member would 


\subsection{Session 2}

\subsubsection{Purpose}

The objective of the second session is to identify the root cause of each member's automatic thoughts and to initiate the move towards modifying these negative beliefs.

\subsubsection{Theme}

The theme of the second session is recognizing core beliefs and its connection with the identified automatic thoughts. There is a direct link on the manner by which automatic thoughts are being acquired from core beliefs as mediated by cognitive distortions.

\subsubsection{Activities}

The facilitator makes a recap of the things that took place during the initial session, specifically on the role of automatic thoughts in the formation of career indecision. Then the facilitator will present the definition of core beliefs and where they can be acquired along with cognitive distortions that would be influential in the crystallization of automatic thoughts. Thereafter, each member will be allowed to think and recognize among the core beliefs that are directly connected with the automatic thoughts that they have identified during the first session. They will then be asked to share their core beliefs while other members are encouraged to interact if they would like to give any comments or feedback about such core beliefs. Cognitive distortions that can be linked to such beliefs will be also identified. The facilitator must make sure that each member has shared their core beliefs and pertinent cognitive distortions.

\subsubsection{Process}

The session is an extension of the self-assessment procedures that were executed during the first session and an educative intervention to promote awareness as regards to the root causes of the members' automatic thoughts. It is the set point of getting a clearer picture the members' perceived difficulties in making career decisions. This will allow them to realize the origin of the concerns they are confronted with.

\subsection{Session 3}

\subsubsection{Purpose}

The aim of this session is to actively engage each member in modifying their identified negative beliefs that they hold about themselves through series of individual activities.

\subsubsection{Theme}

The theme for the third session is recognizing each member's capability to modify their automatic thoughts.

\subsubsection{Activities}

The first activity is an individualized intervention which would allow each member to modify their automatic thoughts. Using series of questions, the facilitator will let the members expose the negative thinking pattern that they have by writing their responses on a sheet of paper. The logic of their negative beliefs will be sought through looking at the evidences justifying the existence of such thoughts. This will serve as the main activity as well for the session. Then processing follows which will zero in on the learning or insights gained from the activity. Each member is allowed to share their learning/insights and to interact with each other in making sense out of the things learned from the activity. Thereafter, members will be asked to read an article about the use of logical questioning in disputing negative thinking patterns which will be used for the next session. 


\subsubsection{Process}

The activities that are carried out for this session would resemble the techniques that are frequently employed in cognitive therapy. Individual cognitive exercise is an essential way to modify the negative thoughts that they hold about themselves. The importance of social support and a feeling of belongingness are partially manifested as well in the processing part given that each member is allowed to share insights and interact with one another to facilitate interpersonal learning, a salient curative factor as enunciated by Irvin Yalom.

\subsection{Session 4}

\subsubsection{Purpose}

The objective of this session is to enable members dispute the negative beliefs that they hold about themselves through dyadic and interactive activities.

\subsubsection{Theme}

The theme for this session is continuously executing strategies that help each member modify automatic thoughts that they have in relation to career decision-making.

\subsubsection{Activities}

After having a recap on the things that took place on the third session, the facilitator asks the members about the articles that they have read about the steps in modifying negative beliefs. Then a behavioral role-play exercise will be facilitated. In this activity, the facilitator will ask the members to work with a partner and let each member identify and dispute negative beliefs of their partners. Since this will be done on pair, each member will undergo the same procedure. Thereafter, the facilitator processes the activity through engaging the members in an interactive discussion that highlights the things and insights that they have gained from participating in the dyadic activity. Lastly, members will be ask to look at specific steps in making specific steps in making career decisions through searching an article in the internet or other scholarly papers.

\subsubsection{Process}

The activities in this session are consistent with the set of strategies employed under the paradigm of cognitive-behavioral therapy. Facilitating an activity that engages each member in the process of disputing their negative career thoughts allows them to gain a deeper understanding in becoming resilient career decision-makers laden with the role of evidence-based and logical thinking patterns. Given that the main activity is done in groups through dyads, the significance of social support in acquiring therapeutic gains is given due importance. This can be seen as well on the interactive discussions that place during the session.

\subsection{Session 5}

\subsubsection{Purpose}

The objective of this session is to keep on exploring ways on how to modify the negative beliefs that they hold about themselves in relation to making career decisions.

\subsubsection{Theme}

The theme of this session is identifying efficient and systematic strategies on how to make career decisions based on evidences.

\subsubsection{Activity}

The members will be asked to share the efficient strategies that they have learned from the homework given 
after having a recap of what has taken place on the fourth session. Then the facilitator encourages members to provide comments or suggestions on the things that will be brought about by the member who will be sharing specific steps to make career decisions. Once in a while, the facilitator employs specific techniques that will let the members gain a more adaptive and neutral way of looking at the process of making career decisions like facilitating, disclosing, linking and the likes. Members are also given the opportunity to express their thoughts about the things that were shared by some members. After the activity, processing of the experiences follows.

\subsubsection{Process}

With the sharing of the things that are learned in the homework, the members are given the opportunity to look at evidences of adaptive behavioral patterns that would characterize proper ways to make career decisions. The manner of processing the dynamics of the group is still consistent with the core principles of Cognitive-Behavioral Therapy. Also, letting each member share what they are thinking about the strategies in making career decisions implies active engagement by members in the group process.

\subsection{Session 6}

\subsubsection{Purpose}

The objective of sixth and last session is to execute a culminating activity where the actions taken every session are cited along with the positive changes that have taken place as a result of the group process. Specific career decision or choices that members come up with will be discussed as well.

\subsubsection{Theme}

The theme for the last session is appraising and summarizing the things that are learned from the previous sessions. Highlighting positive changes, especially on the way they look at things that took place as a result of the group process is a principal matter in this session as well. Particular courses of career actions that each member intends to implement in the future is given due attention.

\subsubsection{Activity}

The facilitator makes a recap of the things that have transpired in the previous session. Then each member is asked to take note of the changes on the way they think about career decision-making and specific career plans in the future as a result of the intervention. Thereafter, each member will share the changes they have experienced along with the plans that they intend to have in relation to his/her career path. That activity serves as a take-off point towards recognizing the role of each member in contributing to the positive changes that they acquired from the group interaction in every session. Members are encouraged to thank and positively appraise one another for the success of the group intervention. Lastly, the facilitator pulls out essential themes and insights that came out of the sharing activities and uses it in continuously empowering each member to have a logical and constructive way to look at the things or events that may be linked to their perceived capabilities to become resilient career decision-makers.

\subsubsection{Process}

The last session is a conceptualized to let each member realize the beneficial effects of having career thinking patterns grounded on logic and evidences. The facilitator recognizes the efforts of each member in attaining the group goals. Letting each member identify the contribution of other members in perpetuating positive changes on their career decision-making capabilities empower them to continuously employ learned strategies in addressing career decision difficulties in the future. Supportive and positive atmosphere for commitment to increased career decidedness is being zeroed in as well given that each member expresses gratitude and positive appraisal of the things they are able to offer in the group. The facilitator encourages members to look at the logic of the circumstances or events that will happen to them and to practically apply 
Datu, J. A.

cognitive and behavioral skills they have acquired in the group process, not just in career decision related issues but on other concerns that will be confronted with.

\section{Strategies to evaluate the group}

\subsection{Career decision profile}

Career Decision Profile is a 16-item inventory devised by Jones (1989) to measure individuals' level of career indecision. The test is administered before and after implementing the intervention to check if the members' career decidedness has improved as evidenced by lower scores in this test.

\subsection{Group appraisal}

The group will be given an opportunity to share their evaluation of the group intervention. Members are encouraged to cite and thank as well the significant contributions of the members who have helped in changing the way they think about making career choices. This would be essential in continuously equipping them with the resources that they would need in dealing with future circumstances that calls for the needs to make occupational decisions.

\subsection{Group Case notes}

These are the personal notes of the facilitator with respect to the group events and processes observed in every session. It is an essential element in the evaluative template of the facilitator as it looks at the progress among the group as a result of the intervention. Group process and events are noted every session to ensure that therapeutic encounters are well-documented. It serves as a basis as well in checking if the group objectives per session are met.

\section{Referral procedures and follow-up}

In view of the fact that this group is not a psychotherapy group as regards to its purpose, the procedures for referral must be in accordance with the usual protocols that are done in individual counseling or psychotherapeutic sessions. Members who would like to be helped in other emotional and psychological issues are encouraged to set appointments with university counselors or psychologists to address their peculiar concerns. Additionally, the group facilitator can also refer members whom he/she perceived to be needing of further assessments or interventions.

\section{References:}

Cox, D. E., \& Harrison, D. W. (2008). Model of anger: Contributions from psychophysiology, neuropsychology and cognitive behavioral perspective. Journal of Brain Structural Functions, 212, 371-385. $<$ http://dx.doi.org/10.1007/s00429-007-0168-7>

Dimakakou, D. S., Mylonas, K., Argyropoulou, K., \& Tampouri, S. (2012). Career decision-making difficulties, dysfunctional thinking and general self-efficacy of university students in Greece. World Journal of Education, 2(1), 117-130.

Fujisawa, D., Nakagawa, A., Tajima, M., Sado, M., Kikuchi, T., Hanaoka, M., \& Ono, Y. (2010). Cognitive behavioral therapy for depression among adults in Japanese clinical settings: A single-group study. BMC Research Notes, 3, 1-7. <http://dx.doi.org/10.1186/1756-0500-3-160>

Granvold, D. K. (1994). Cognitive and behavioral treatment: Methods and applications. Pacific Grove, CA: Brooks/Cole Publishing.

Hamblen, J. L., Norris, F. H., Pietruszkiewicz, S., Gibson, L. E., Naturale, A., \& Louis, C. (2009). Cognitive behavioral therapy for post-disaster stress: A community based treatment for survivors of Hurricane 
Katrina. Administration Policy of Mental Health, 36, 206-214.

$<$ http://dx.doi.org/10.1007/s10488-009-0213-3>

Marcotte, D., Levesque, N., \& Fortin. L. (2006). Variations of cognitive distortions and school performance in depressed and non-depressed high school students: A two-year longitudinal study. Cognitive Therapy Research, 30, 211-225. <http://dx.doi.org/10.1007/s10608-006-9020-2>

Sica, C., Taylor, S., Arrindell, W. A., \& Sanavio, E. (2006). A cross-cultural test of the cognitive theory of obsessions and compulsions: A comparison of Greek, Italian, and American individuals: A preliminary study. Cognitive Therapy Research, 30, 585-597. <http://dx.doi.org/10.1007/s10608-006-9078-x> 
Datu, J. A. 